\title{
139 ADVANCE CARE PLANNING AS A DYNAMIC PROCESS: A DESCRIPTIVE 1 YEAR REVIEW OF CHANGES IN ACP PLANS IN TAN TOCK SENG HOSPITAL
}

$\mathrm{R} \mathrm{Ng},{ }^{1} \mathrm{R}$ Chong, ${ }^{2} \mathrm{H} \mathrm{Chau},{ }^{2} \mathrm{C} \mathrm{H} \mathrm{Poi,}{ }^{1}$ J Eng Ramdat, ${ }^{1}$ Y Z May, ${ }^{2}$ A Loke, ${ }^{2}$ H Y Wu ${ }^{1}{ }^{1}$ Tan Tock Seng Hospital, Palliative Medicine; ${ }^{2}$ Tan Tock Seng Hospital, Operations Community, Singapore

10.1136/bmjspcare-2013-000491.139

Background Advance care planning (ACP) is a process and people can change their minds about treatment preferences when goals of care change. The ACP project team in Tan Tock Seng Hospital continually audits ACP outcomes including any change in ACP plans.

Aim To examine rate of change in ACP plans and factors that may have contributed to changes in ACP plans.

Methods ACP outcomes for readmissions and death were analysed from retrospective audit of paper and electronic medical records.

Results Between October 2011 to December 2012, a total of 154 Preferred Plan of Care (PPC), a POLST-type ACP were completed with patients and/ or their next-of-kin (NOK). Out of these 154 PPCs, 5 ACP plans were revised. Time lapse between creation of first and second ACP plan ranged from 3 to 91 days. One patient had decision making capacity at first and second ACP documentation, while 4 other patients had no decision making capacity at both ACP documentation. four revisions were initiated by doctors whilst one was initiated by the substitute decision maker. Two ACP plans were changed after further clarifications with NOK whilst three revisions took place when there were changes in the patients' medical condition or after trial of medical intervention. Three of the patients have since passed away.

Discussion The number of changed ACP plans is a small fraction of the total completed. Doctors were active in continual exploration of ACP plans.

Conclusion In our cohort, ACP plans remain relatively stable. ACP is a dynamic process that need a culture of review and re-exploration of care preferences in order to be responsive to changes in medical condition and care preferences. 